\title{
La Celestina en escenarios argentinos: observaciones comparatistas sobre las adaptaciones de Jorge Goldenberg (1993) y Daniel Suárez Marzal (2007)
}

\author{
Jorge Dubatti \\ Universidad de Buenos Aires
}

La Celestina es un texto incluido en el canon escolar (escuela secundaria) de la Argentina y por lo tanto es conocido tempranamente por un lectorado masivo. Las ediciones disponibles - empleadas en las aulas-ofrecen el texto completo, aunque actualizado en su dimensión lingüística. Pero ¿cómo llega La Celestina a los argentinos a través de los escenarios?

La dramaturgia de adaptación es una de las modalidades más frecuentes de vinculación de los escenarios argentinos con el teatro extranjero, y especialmente con el teatro de épocas lejanas. La adaptación teatral consiste en la reescritura teatral (dramática y/o escénica) de un texto-fuente (teatral o no) previo, reconocible y declarado, versión elaborada con la voluntad de aprovechar la entidad poética del texto-fuente para implementar sobre ella cambios de diferente calidad y cantidad. El reconocimiento de la categoría dramaturgia de adaptación resulta una de las conquistas más valiosas de la disciplina Teatro Comparado y se conecta además con la ampliación del concepto de texto dramático consolidada en la Argentina de los años de Postdictadura, relacionable a su vez con las categorías de dramaturgia de dirección y de actuación. ${ }^{1}$

En el caso de La Celestina, cuya primera edición es de 1499, la extensión de sus 16 autos (poco más tarde 21) y su liminalidad con la «novela dialogada» la tornan prácticamente "irrepresentable» en términos de teatralidad contemporánea; este rasgo de su poética original exige inexora- 
blemente la mediación de la adaptación para favorecer su llegada a los escenarios actuales.

Analizaremos algunos de los lineamientos principales del trabajo de dramaturgia de adaptación de La Celestina realizado por Daniel Suárez Marzal en 2007 (Complejo Teatral de Buenos Aires, Teatro Regio; reposición en 2008 en el Teatro San Martín) y realizaremos algunos enlaces comparativos con la versión de La Celestina concretada por Jorge Goldenberg en 1993, también destinada al circuito del teatro oficial porteño (Teatro San Martín). ${ }^{2}$ Ambas coinciden en su entidad de adaptación poética (no genérica): tanto el texto-fuente como la adaptación son textos teatrales, no hay cambio genérico sino modificaciones dentro de las respectivas poéticas teatrales (el conjunto de procedimientos que, por selección y combinación, generan un determinado efecto teatral y portan una ideología estética). A la vez se trata de versiones muy diferentes pero que, sin embargo, desde poéticas diversas, preservan una relación celebratoria con el texto clásico español. La puesta en escena de la adaptación de Jorge Goldenberg estuvo a cargo de Osvaldo Bonet; ${ }^{3}$ Daniel Suárez Marzal dirigió el montaje de su propia versión. ${ }^{4}$ Recordemos que Suárez Marzal cuenta con una valiosa trayectoria en la puesta en escena de clásicos del teatro español (Numancia, ${ }^{5}$ La vida es sueño, El perro del hortelano, entre otras) y estuvo al frente del Instituto de Teatro de Sevilla.

No nos referiremos a los textos escénicos de los acontecimientos teatrales (funciones, representaciones de la obra) sino que limitaremos nuestras observaciones a los textos dramáticos disponibles, inédito el de Goldenberg (texto mecanografiado, 113 páginas, fechado en 1992, en consecuencia de estatuto pre-escénico, ${ }^{6}$ que se conserva en el Archivo del Centro de Documentación del Complejo Teatral de Buenos Aires), y publicado el de Suárez Marzal (también de estatuto pre-escénico). Por razones de extensión, nos centraremos en observaciones sobre el nivel de la historia y el nivel textual (Mieke Bal, 1998).

El texto dramático de Suárez Marzal sobresale por su operación de reducción radical del texto-fuente; baste señalar que Suárez Marzal trabaja

2.- Para un desarrollo detenido en la versión de Jorge Goldenberg, véase nuestro "Jorge Goldenberg y la dramaturgia de adaptación: notas sobre La Celestina (1993)», en Goldenberg 2008 (en prensa).

3.- La versión dirigida por Bonet incluyó en los roles principales a Graciela Araujo (Celestina), Juan Palomino (Calisto) y Laura Novoa (Melibea).

4.- Los tres actores: Elena Tasisto (Celestina), Sergio Surraco (Calisto) y Julieta Díaz (Melibea), en escena junto a los cantantes Pehuén Díaz Bruno y Nicolás Bernazzani y el músico Miguel de Olaso.

5.- Hemos estudiado en otra ocasión su adaptación de Numancia de Cervantes (véase Dubatti 2006).

6.- Llamamos texto dramático pre-escénico a una clase de texto dramático literario dotado de virtualidad escénica, escrito a priori, antes e independientemente de la escena, que guarda un vínculo transitivo con la puesta [del texto] en escena. 
con la representación escénica de sólo tres personajes (Calisto, Melibea, Celestina), ${ }^{7}$ se centra en la historia de amor (en su dimensión gozosa y trágica) y compone 16 escenas —en su mayoría breves - que remiten a los siguientes autos y escenas ${ }^{8}$ de La Celestina:

\begin{tabular}{|c|c|}
\hline $\begin{array}{l}\text { Texto adaptación de Suárez } \\
\text { Marzal }\end{array}$ & $\begin{array}{l}\text { Texto-fuente de Fernando de } \\
\text { Rojas }\end{array}$ \\
\hline $\begin{array}{l}\text { Escena I (encuentro de Calisto y } \\
\text { Melibea en el jardín) }\end{array}$ & $\begin{array}{l}\text { Primer auto, Escena } 1 \text { (encuentro } \\
\text { de Calisto y Melibea en el jardín) }\end{array}$ \\
\hline $\begin{array}{l}\text { Escena II (Calisto en su casa, solo, } \\
\text { expresa su amor por Melibea; in- } \\
\text { cluye una canción) }\end{array}$ & $\begin{array}{l}\text { Primer auto, Escena } 2 \text { (Calisto ha- } \\
\text { bla con Sempronio) }\end{array}$ \\
\hline $\begin{array}{l}\text { Escena III (en una calle, Celestina } \\
\text { busca a Calisto; Celestina se pre- } \\
\text { senta a sí misma y acuerda con } \\
\text { Calisto su servicio) }\end{array}$ & $\begin{array}{l}\text { Primer auto, Escenas } 12 \text { y } 14 \text { (diá- } \\
\text { logo de Calisto y Celestina), más } \\
\text { intercalaciones de fragmentos de } \\
\text { otras escenas del mismo auto }\end{array}$ \\
\hline $\begin{array}{l}\text { Escena Iv (Celestina sola, por la } \\
\text { calle, reflexiona sobre su encargo } \\
\text { mientras camina hacia la casa de } \\
\text { Melibea; llegando dice ver salir } \\
\text { a la madre de Melibea, quien le } \\
\text { deja así el campo de acción libre) }\end{array}$ & $\begin{array}{l}\text { Cuarto auto, Escena } 1 \text { ("Celesti- } \\
\text { na, andando por el camino, habla } \\
\text { consigo misma hasta llegar a la } \\
\text { puerta de Pleberio») }\end{array}$ \\
\hline $\begin{array}{l}\text { Escena v (Encuentro de Celestina } \\
\text { y Melibea en casa de ésta última; } \\
\text { el hilado, la oración por el dolor } \\
\text { de muelas y el cordón) }\end{array}$ & $\begin{array}{l}\text { Cuarto auto, Escena } 5 \text { (diálogo de } \\
\text { Celestina y Melibea, con la pre- } \\
\text { sencia de Lucrecia) }\end{array}$ \\
\hline $\begin{array}{l}\text { Escena VI («Noche, un descampa- } \\
\text { do siniestro», Celestina sola rea- } \\
\text { liza el conjuro a Plutón; incluye } \\
\text { la "Copla a la Hermosa Rapada» } \\
\text { y referencias a la ingratitud de los } \\
\text { poderosos; sale en busca de Ca- } \\
\text { listo) }\end{array}$ & $\begin{array}{l}\text { Tercer auto, Escena } 3 \text { (conjuro a } \\
\text { Plutón) } \\
\text { Primer auto, Escena } 11 \text { (fragmen- } \\
\text { tos del diálogo de Celestina con } \\
\text { Pármeno) }\end{array}$ \\
\hline
\end{tabular}

7.- No se hace referencia a los músicos en el texto dramático pre-escénico.

8. - Llamamos «escenas» a las divisiones internas de los autos a través de la marca tipográfica de una barra horizontal (edición del texto-fuente de Bruno Mario Damiani, Cátedra, 1980). 
Escena vII (Celestina visita a Calisto en su casa, le entrega el cordón, le promete pronto encuentro con Melibea, se lleva consigo el cordón para usarlo en un conjuro y se va)

Escena vill (Melibea, sola, en su casa, confiesa su amor por Calisto en un monólogo; incluye una canción)

Escena IX (encuentro de Melibea y Celestina en su casa, Melibea le confiesa su amor, pide los servicios de Celestina, arreglan encuentro con Calisto a la medianoche)

Escena $x$ (encuentro de Celestina y Calisto en la calle, información para el encuentro con Melibea a medianoche, Calisto le entrega a celestina una cadena de oro)

Escena XI (encuentro nocturno de Calisto y Melibea, frente e interior de la casa de Melibea, portón entreabierto; Calisto viene de la calle, Melibea se asoma del interior; declaración de amor mutuo; arreglan la cita en el huerto para la medianoche siguiente)

Escena XII (a la mañana siguiente, víspera de los amantes en escena paralela: a la izquierda Calisto en su alcoba; a la derecha Melibea en el balcón de la torre de su casa; incluye canciones)

Escena XIII (encuentro de Calisto y Melibea en el huerto, medianoche; consumación del amor)
Sexto auto, Escena única (dialogo de Calisto y Celestina con la presencia de los criados Sempronio y Pármeno)

Décimo auto, Escena 1 («... está hablando Melibea consigo misma»)

Décimo auto, escenas 2 y 3 (diálogo Celestina y Melibea, expulsan a Lucrecia)

Onceno auto, Escena 1 (diálogo de Celestina y Calisto, con la presencia de Sempronio y Pármeno)

Doceno auto, Escenas 2 y 3 (encuentro Calisto y Melibea, con la presencia de los criados Sempronio, Pármeno y Lucrecia)

Treceno auto, Escena 1 (Calisto con Tristán y Sosia)

Catorceno auto, Escenas 1 y 3 (Melibea con Lucrecia, Melibea con Calisto)

Catorceno auto, Escenas 2 y 4 


\begin{tabular}{|l|l|}
\hline $\begin{array}{l}\text { Escena XIV (esa madrugada, en la } \\
\text { calle, Calisto vuelve a su casa y } \\
\text { encuentra a Celestina, que viene } \\
\text { herida de muerte por su pelea con }\end{array}$ & $\begin{array}{l}\text { Catorceno auto, Escena } 9 \text { (monó- } \\
\text { logo de Calisto) } \\
\text { Doceno auto, Escena } 7 \text { (pelea de } \\
\text { Celestina con Sempronio y Pár- } \\
\begin{array}{l}\text { Sempronio y Pármeno, noticia } \\
\text { de lo que ha sucedido y de que } \\
\text { los criados han sido prendidos y } \\
\text { muertos) }\end{array}\end{array}$ \\
\hline $\begin{array}{l}\text { Escena Xv (en el huerto de Meli- } \\
\text { bea, a la medianoche, Melibea } \\
\text { canta, llega Calisto, se encuen- } \\
\text { tran, pero al escuchar alboroto del } \\
\text { otro lado de la pared Calisto salta } \\
\text { y es asesinado en una emboscada, } \\
\text { muere «atravesado») }\end{array}$ & $\begin{array}{l}\text { Decimonono auto, todas las es- } \\
\text { traición de Areúsa, Calisto llega } \\
\text { al huerto, Lucrecia y Melibea can- } \\
\text { Calisto, accidente fatal («su cabe- } \\
\text { za está en tres partes») }\end{array}$ \\
\hline $\begin{array}{l}\text { Escena xvi (en la torre de la casa } \\
\text { de Melibea, ésta sola, monólogo } \\
\text { y suicidio) }\end{array}$ & $\begin{array}{l}\text { Veinteno auto, Escenas } 3 \text { y } 4 \text { (Me- } \\
\text { libea se suicida luego de explicar } \\
\text { lo sucedido a su padre) }\end{array}$ \\
\hline
\end{tabular}

De este cuadro se desprenden muchas de las operaciones que Suárez Marzal realiza sobre la historia del texto-fuente. Sugerimos al lector considerar la columna de la adaptación en su recorrido vertical para advertir el efecto de linealidad y simplificación de la intriga. Por otra parte, la lectura vertical de la columna del texto de Rojas, para observar los saltos y la deliberada exclusión de vastas zonas del texto-fuente. Ya se señaló la ausencia escénica de numerosos personajes esenciales (los criados, las prostitutas, Pleberio y Alisa, Centurio, Crito, etc). Señalemos otras operaciones destacables:

1.- Daniel Suárez Marzal descarta autos completos desde el punto de vista de la historia y sus situaciones (en algunos casos sólo toma de ellos expresiones textuales que reinserta en otras situaciones de enunciación, como es el caso del retrato de Melibea en boca de Calisto, o del retrato de Celestina en boca de Sempronio). Se centra en las situaciones de los autos I, III, IV, VI, X, XI, XII, XIII, XIV, XIX Y XX.

2.- adelgaza la gradación de conflictos, desaparecen las intrigas paralelas, se gana en linealidad. Lo que en el texto de Rojas implica mediaciones, dilaciones, intermediarios y desvíos, en la adaptación se vuelve lineal y directo.

3.- cambia situaciones: encuentros en las calles desplazan las escenas de interiores, Celestina informa que ve salir a la madre de Melibea, la víspera de los amantes es jugada en escena simultánea a través del 
paralelo de los dos ámbitos, Calisto no muere en un accidente de caída, etc.

4.- reorganiza el orden y la causalidad de las escenas: el conjuro a Plutón es posterior a la visita de Celestina a Melibea (en consecuencia se refiere en el texto de Suárez Marzal al hilado como algo ya comprado por Melibea; véase enseguida el análisis del fragmento); la información de la muerte de Celestina, Sempronio y Pármeno llega después de la consumación del amor (que se concreta entonces en ignorancia de los sucesos nefastos).

5.- multiplica las situaciones de canto, favoreciendo la transformación de La Celestina en teatro musical (hecho que acentuará el texto escénico con la inclusión de cantantes y músico). Recuérdese que Suárez Marzal es un destacado régisseur de ópera.

6.- incluye textos nuevos, como la canción inicial de Calisto o la «Copla de la Hermosa Rapada», o parlamentos que permiten referir situaciones no presenciadas escénicamente a través de relatos (la escena de la muerte de Celestina a manos de Sempronio y Pármeno se transforma aquí en el relato que la propia Celestina hace mientras agoniza).

7.- en cuanto a las operaciones lingüísticas, resulta valioso confrontar el conjuro a Plutón en el texto-fuente y en la adaptación (nos abstraeremos de las didascalias, abundantes en Suárez Marzal, para centrarnos en el habla del personaje): 


\begin{tabular}{|c|c|}
\hline Tercer auto, Escena 3 & Escena VI \\
\hline $\begin{array}{l}\text { CELESTINA: Conjúrote, triste Plutón, } \\
\text { señor de la profundidad infernal, empe- } \\
\text { rador de la corte dañada, capitán sober- } \\
\text { bio de los condenados ángeles, señor de } \\
\text { los sulfúreos fuegos que los hirvientes } \\
\text { étnicos montes manan, gobernador y } \\
\text { veedor de los tormentos y atormentado- } \\
\text { res de las pecadoras ánimas, regidor de } \\
\text { las tres furias, Tesífone, Megera y Aleto, } \\
\text { administrador de todas las cosas negras } \\
\text { del reino, de Estigie y Dite, con todas } \\
\text { sus lagunas y sombras infernales y liti- } \\
\text { gioso caos, mantenedor de las volantes } \\
\text { harpías, con toda la otra compañía de } \\
\text { espantables y pavorosas hidras. }\end{array}$ & $\begin{array}{l}\text { CELESTINA: ¡Te conjuro, triste Plutón, } \\
\text { señor de la profundidad infernal, empera- } \\
\text { dor de la corte dañada, capitán soberbio } \\
\text { de los ángeles condenados, señor de los } \\
\text { fuegos sulfurosos que manan de la tierra, } \\
\text { gobernador de los tormentos y de los } \\
\text { atormentadores de las ánimas pecadoras, } \\
\text { regidor de las tres furias, Tesífone, Me- } \\
\text { gera y Aleto, administrador de todas las } \\
\text { cosas negras del reino, de Estige y Dite, } \\
\text { con todas sus lagunas y sombras inferna- } \\
\text { les y el litigioso Caos, mantenedor de las } \\
\text { Harpías voladoras, con su compañía de } \\
\text { las espantables y pavorosas Hidras! }\end{array}$ \\
\hline $\begin{array}{l}\text { Yo, Celestina, tu más conocida cliéntula, } \\
\text { te conjuro por la virtud y fuerza de estas } \\
\text { bermejas letras, por la sangre de aquella } \\
\text { nocturna ave con que están escritas, por } \\
\text { la gravedad de aquestos nombres y sig- } \\
\text { nos que en este papel se contienen, por } \\
\text { la áspera ponzoña de las víboras de que } \\
\text { este aceite fue hecho, con el cual unto } \\
\text { este hilado; vengas sin tardanza a obe- } \\
\text { decer mi voluntad y en ello te envuel- } \\
\text { vas y con ello estés sin un momento te } \\
\text { partir, hasta que Melibea, con aparejada } \\
\text { oportunidad que haya, lo compre y con } \\
\text { ello de tal manera quede enredada, que } \\
\text { cuanto más lo mirare, tanto más su cora- } \\
\text { zón se ablande a conceder mi petición, y } \\
\text { se le abras y lastimes del crudo y fuerte } \\
\text { amor de Calisto; tanto que, despedida } \\
\text { toda honestidad, se descubra a mí y me } \\
\text { galardone mis pasos y mensaje; y esto } \\
\text { hecho, pide y demanda de mí a tu vo- } \\
\text { luntad. }\end{array}$ & $\begin{array}{l}\text { Yo, Celestina, tu clientucha más conoci- } \\
\text { da, te conjuro por la verdad de estas letras } \\
\text { bermejas escritas con la sangre del mur- } \\
\text { ciélago al que le corté primero las uñas, } \\
\text { por la áspera ponzoña con que fue hecho } \\
\text { ese aceite con el cual unté los hilados que } \\
\text { compró Melibea, a que vengas sin tar- } \\
\text { danza y te envuelvas con esos hilos, que } \\
\text { otros iguales te he reservado, para que } \\
\text { cuanto más ella mire mis hilados, tanto } \\
\text { más su corazón se ablande. }\end{array}$ \\
\hline $\begin{array}{l}\text { Si no lo haces con presto movimiento, } \\
\text { ternásme por capital enemiga; heriré con } \\
\text { luz tus cárceles tristes y escuras; acusa- } \\
\text { ré cruelmente tus continuas mentiras; } \\
\text { apremiaré con mis ásperas palabras tu } \\
\text { horrible nombre. } \\
\text { Y otra y otra vez te conjuro; y así con- } \\
\text { fiando en mi mucho poder, me parto } \\
\text { para allá con mi hilado, donde creo te } \\
\text { llevo ya envuelto. }\end{array}$ & $\begin{array}{l}\text { Si no lo cumplieses rápidamente me ten- } \\
\text { drás por enemiga capital, heriré con luz } \\
\text { tus cárceles tristes y oscuras, acusaré } \\
\text { cruelmente tus continuas mentiras, y lle- } \\
\text { naré, por donde vaya, el aire con insultos } \\
\text { a tu nombre horrible. }\end{array}$ \\
\hline
\end{tabular}


Hemos señalado antes que Suárez Marzal modifica la situación del conjuro al ubicarla luego de la entrevista con Melibea. Desde el punto de vista estrictamente lingüístico, reduce el parlamento del conjuro (la última oración no aparece), actualiza la lengua (escuras/oscuras; ternásme/me tendrás), altera la sintaxis y la puntuación, agrega signos de admiración, modifica el léxico (sulfúreos/sulfurosos; volantes/voladoras) y el sentido (cliéntula/clientucha; virtud/verdad; crudo y fuerte amorl fuerte dolor y deseo), inserta textos provenientes de otros pasajes (al que le corté primero las uñas), cambia la posición de los pronombres y los adjetivos, explicita expresiones ambiguas (la nocturna ave del conjuro se transforma en murciélago).

La adaptación de Jorge Goldenberg se diferencia de la de Suárez Marzal por rasgos evidentes:

- se trata de un texto dramático muchísimo más extenso, que -como manifiestan las marcas manuscritas de trabajo sobre el original mecanografiado que se conserva- debió ser acortado para la puesta en escena.

- posee un sistema de personajes actualizados en escena más amplio, que incluye a Pleberio, Sempronio, Pármeno, Elicia, Alisa, Areúsa, y que en consecuencia apuesta menos a la linealidad de la historia de amor que a la escenificación del universo-Celestina

- otorga especial valor a la estructura de los diálogos del texto-fuente, tan celebrados por la crítica.

- enmarca la historia con el planto de Pleberio, que abre el texto y lo cierra.

- el mundo que Fernando de Rojas deja en el subtexto de algunas situaciones, Goldenberg lo explicita: por ejemplo, en la Escena 2, que reenvía al primer encuentro de Calisto y Melibea, Goldenberg propone en didascalia: «Se oye -muy simplificado- el aleteo de un ave. Enseguida, una luz muy puntual ilumina un halcón que atraviesa el espacio y va a posarse sobre una rama muy alta. Tras un tiempo, se oyen unos pasos a la carrera [Calisto], acompañados de sonido de espuelas» (página 1).

- Goldenberg no apuesta en su texto a la innovación de la poética del teatro musical (tan apreciada por Suárez Marzal, frecuente en su teatro), sino a la valorización de la literatura de Rojas, a la que busca poner en primer plano (aunque la puesta de Bonet incluiría más tarde música de Jorge Valcarcel).

Contrastadas, la adaptación de Suárez Marzal se manifiesta minimalista frente al despliegue textual de Goldenberg. Si bien la dramaturgia de Suárez Marzal parte de un proyecto individual, hay que resaltar su complementariedad con las posibilidades actuales de producción en el 
teatro oficial, que favorece el minimalismo económico, la austeridad, el bajo presupuesto.

Desde el punto de vista de las alteraciones cualitativas del texto-fuente, la versión de Goldenberg se encuadra en una adaptación clásica: sigue muy de cerca las instrucciones implícitas y explícitas de la poética del textofuente, implementando respecto de ellas sólo cambios escasamente relevantes y concentrando su singularidad en la lectura de los lugares de indeterminación (en complementariedad con las instrucciones implícitas/ explícitas del texto). Goldenberg busca establecer un puente (principalmente morfológico) entre la temporalidad poética del texto-fuente (anclada en su historicidad, anacrónica respecto del presente) y la temporalidad poética del teatro actual (sincrónica), a partir del reconocimiento de la vigencia o actualidad de Rojas.

Por el contrario, las alteraciones cualitativas en la versión de Suárez Marzal son más profundas, y encuadra en la adaptación estilizante: si bien sigue de cerca la poética del texto-fuente, incrementa considerablemente la alteración cualitativa e imprime modificaciones a la poética que profundizan su novedad y singularidad. Los cambios recorren todos los niveles pero no llegan a constituirse en reversiones o inversiones de la poética del texto-fuente. La de Suárez Marzal es una adaptación estilizante formal: los cambios más relevantes pasan por los procedimientos morfológicos de la poética, aunque también afectan decisivamente a los componentes esenciales de la fábula y transforman con nuevos matices la semántica resultante.

Si bien señalamos arriba que se adapta o rescribe porque no hay posibilidad teatral de no hacerlo, creemos que tanto Suárez Marzal como Goldenberg respetan la autoría y la entidad del texto con distintas funciones, intenciones u objetivos:

- volver sobre la monumentalidad poética de la obra: se considera que el texto-fuente es una obra maestra, digna de ser conocida y disfrutada en el presente, porque acaso es más significativa, vigente e incisiva que mucha de la producción actual; a través de la dramaturgia de adaptación se invita a recordar y homenajear el texto-fuente.

- la enseñanza o divulgación didáctica: la versión contribuye a difundir este gran texto a través del formato teatral, y de esta manera se cumple con uno de los objetivos fundamentales del teatro oficial, la educación.

- el autoconocimiento: se emprende la adaptación con la conciencia de que la reescritura pone en evidencia la identidad del que re-escribe, define la diferencia de su personalidad, de su tiempo, de su territorialidad.

- la resiliencia o adaptación del texto-fuente a las condiciones de producción locales (limitaciones económicas, de elenco, de espacio, etc.). 
- el goce de jugar, experimentar o dialogar con el otro texto, de disfrutar los hallazgos de su estructura dialogando poéticamente con él. Kado Kostzer (2008) llama a sus versiones «diversiones», puro placer de juego. ${ }^{9}$

De esta manera La Celestina se abre camino, desde el pasado español, para reanudar su vínculo con los espectadores argentinos. Jorge Goldenberg y Daniel Suárez Marzal, más allá de los cambios de reescritura, preservan su función de intermediarios que hacen posible ese encuentro.

\section{Bibliografía}

BAL, Mieke, 1998, Teoría de la narrativa (Una introducción a la narratología), Madrid, Cátedra.

DUBATTI, Jorge, 2006, «Numancia en la Argentina: observaciones comparatistas sobre las versiones escénicas dirigidas por Jorge Petraglia y Daniel Suárez Marzal», en El Quijote en Buenos Aires. Lecturas cervantinas en el cuarto centenario, Alicia Parodi, Julia D'Onofrio y Juan Diego Vila (eds.), Universidad de Buenos Aires, Instituto de Filología y Literaturas Hispánicas "Dr. Amado Alonso", Facultad de Filosofía y Letras y Asociación de Cervantistas, 2006, pp. 859-866.

—, 2008a, «Textos dramáticos y acontecimiento teatral», Cartografía teatral. Introducción al Teatro Comparado, Buenos Aires, Atuel, Cap. IV, pp. 135-171.

—, 2008b, «Jorge Goldenberg y la dramaturgia de adaptación: notas sobre La Celestina (1993)", en Goldenberg 2008 (en prensa).

GoldenberG, Jorge, 1992, La Celestina, texto dramático inédito, archivo del Centro de Documentación Teatral del Complejo Teatral Buenos Aires, 113 páginas.

-, 2005, Teatro completo 1, Buenos Aires, Colihue.

-, 2008, Teatro completo 2, Buenos Aires, Colihue (en prensa).

Kostzer, Kado, y Sergio García-Ramírez, 2008, Versiones y diversiones. Shakespeare, Molière y Sheridan, recreados, Buenos Aires, Colihue.

Rojas, Fernando de, 1980, La Celestina, edición de Bruno Mario Damiani, Madrid, Cátedra.

Salvador Miguel, Nicasio, 2004, "La Celestina», en Javier Huerta Calvo, dir., Historia del teatro español, Madrid, Gredos, tomo I, pp. 137-167. SuÁrez Marzal, Daniel, 2007, Versión de La Celestina de Fernando de Rojas, Buenos Aires, Complejo Teatral de Buenos Aires y Editorial Losada.

9.- Diversión, por otra parte, es una palabra muy adecuada para definir la operación de desvío y fuga, salirse del cauce trazado por el texto-fuente. 


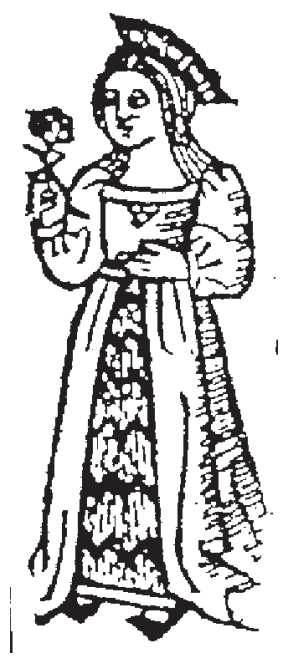


DubaTtI, Jorge, "La Celestina en escenarios argentinos: observaciones comparatistas sobre las adaptaciones de Jorge Goldenberg (1993) y Daniel Suárez Marzal (2007)», Celestinesca, 33 (2009), pp. 59-68.

\section{RESUMEN}

Este trabajo de teatro comparado propone el análisis de La Celestina a partir de las relaciones entre Teatro Argentino Contemporáneo y Teatro Medieval. La indagación central consiste en cómo la reescritura dramática introduce cambios en los textos medievales para su representación fuera de sus contextos originales, en Buenos Aires, en los siglos XX-XXI, desde nuevas concepciones de las poéticas teatrales y tomando en cuenta que La Celestina forma parte del canon escolar de la educación oficial en la Argentina. Primero se realizan observaciones sobre La Celestina adaptada para la puesta en escena por el director Daniel Suárez Marzal (2007, Complejo Teatral de Buenos Aires, Teatro Regio); luego, se concreta el análisis comparativo (aspectos de la estructura narrativa y el nivel textual) con otra reescritura a cargo del dramaturgo Jorge Goldenberg (1993, Teatro Municipal San Martín).

palabras Clave: La Celestina, Teatro Comparado, Teatro Argentino Contemporáneo, reescritura dramática, estructura narrativa, nivel textual.

\section{ABSTRACT}

This article proposes a comparative study about La Celestina and the relations between Contemporary Argentine Theatre and Medieval Theatre. The principal question is: how dramatic rewriting introduces changes in medieval texts for their representations out of their original context, in Buenos Aires, in the XX-XXI Centuries, from new conceptions of theatralical poetics and considering that $L a$ Celestina is included in the scholar canon of official education in Argentina. First, it is considered La Celestina performance-adapted by the director Daniel Suárez Marzal (2007, Complejo Teatral de Buenos Aires, Teatro Regio); then, it takes place the comparative analysis (some aspects of the narrative structure and textual level) with another rewriting by the dramatist Jorge Goldenberg (1993, Teatro Municipal San Martín).

KeY Words: Celestina, Comparative Theatre, Contemporary Argentine Theatre, dramatic rewriting, narrative structure, textual leve. 